\title{
A study of anticipatory non-autonomous systems
}

Book or Report Section

Accepted Version

Hayashi, Y., Spencer, M. and Nasuto, S. (2014) A study of anticipatory non-autonomous systems. In: Awareness Science and Technology and Ubi-Media Computing (iCAST-UMEDIA), 2013 International Joint Conference on. IEEE, pp. 316-318. doi: https://doi.org/10.1109/ICAwST.2013.6765456 Available at http://centaur.reading.ac.uk/37407/

It is advisable to refer to the publisher's version if you intend to cite from the work. See Guidance on citing.

To link to this article DOI: http://dx.doi.org/10.1109/ICAwST.2013.6765456

Publisher: IEEE

All outputs in CentAUR are protected by Intellectual Property Rights law, including copyright law. Copyright and IPR is retained by the creators or other copyright holders. Terms and conditions for use of this material are defined in the End User Agreement. 


\section{www.reading.ac.uk/centaur}

\section{CentAUR}

Central Archive at the University of Reading

Reading's research outputs online 


\title{
A Study of Anticipatory Non-Autonomous Systems
}

\author{
Yoshikatsu Hayashi, Matthew C. Spencer, Slawomir J. Nasuto \\ Brain Embodiment Lab, School of Systems Engineering \\ University of Reading, \\ PO Box 225, Whiteknights, \\ Reading RG6 6AY, UK \\ Website: http://bel.reading.ac.uk/
}

\section{INTRODUCTION}

Rhythms are manifested ubiquitously in dynamical biological processes. These fundamental processes which are necessary for the survival of living organisms include metabolism, breathing, heart beat, and, above all, the circadian rhythm coupled to the diurnal cycle. Thus, in mathematical biology, biological processes are often represented as linear or nonlinear oscillators. In the framework of nonlinear and dissipative systems (ie. the flow of energy, substances, or sensory information), they generate stable internal oscillations as a response to environmental input and, in turn, utilise such output as a means of coupling with the environment.

In the context of human behaviour, [1], [2] have reported instances where body motion may occur prior to an external reference signal in hand tracking and finger tapping tasks. Human subjects are also able to generate motion preceding a periodic target signal [3]. These phenomena indicate that the human sensory-motor system may operate in an anticipatory rather than a reactive mode, when the target motion is relatively fast. Such an anticipatory mode is always accompanied by the generation of a rhythmic component in human motion [4], [5].

In fact, the ability to anticipate future input seems to underpin much cognitive processing. A major advantage of such anticipatory brain mechanisms is that they allow cognitive agents to respond adequately to fast input signals. In addition, the assumption that feedback delays are threats to successful control, being regarded as an unfortunate feature of biological systems, seems to also favour the anticipation hypothesis. Thus, a fundamental question in cognitive neuroscience is what mechanisms might give rise to such signal processing capabilities.

The most common form of postulated anticipatory functionality, so called weak anticipation, requires an explicit internal model, a forward model, simulating the possible outcomes of the external system.

On the other hand, strong anticipation arises from the system itself, via a set of mechanisms embedded in the primary mode of functioning, and without recourse to explicit internal models or representations.

Strong anticipation has been recently proposed as a mechanism of interaction in dynamical systems able to bring about predictions of future states of a master system by a slave system which lacks an explicit model of the master [6]. Interestingly, it is the presence of a delay in the coupling that is a necessary condition to achieve the slave's anticipation of the master's dynamics. The positive, productive status of the delayed feedback should be emphasized in contrast to the weak anticipation, as it may have very important implications for our understanding of how cognitive systems can predict future outcomes during many crucial tasks.

The literature on strong anticipation has mostly concentrated on models of autonomous systems. In this article, we will present work on the anticipatory characteristics of nonautonomous dynamical systems.

\section{Strong Anticipation}

The strong anticipation paradigm was identified by Voss in the general framework of autonomous dynamical systems [7], [8]:

$$
\begin{aligned}
\dot{x} & =f(x) \\
\dot{y} & =f(y)+k\left(x-y_{\tau}\right),
\end{aligned}
$$

where $f(\cdot)$ describes the internal dynamics of the state variables of both the master, $x$, and the slave, $y$, systems. Note that both systems have an identical dynamics. The second term in the slave system is a coupling term which drives the slave to be synchronised with the master system. The term $y_{\tau}$ takes into account the feedback-delay in the slave referring to its own state, namely $y(t-\tau)$.

The strong anticipation is simply identified by the manifold $y(t)=x(t+\tau)$, i.e., the slave system is anticipating the future state of the master system. It is counterintuitive to see that the feed-back delay in the slave is a key to generate the anticipatory synchronisation in the autonomous system.

Regarding non-autonomous systems, anticipatory synchronisation can be extended to:

$$
\begin{aligned}
& \dot{x}=f(x)+I(t) \\
& \dot{y}=f(y)+I(t)+k\left(x-y_{\tau}\right),
\end{aligned}
$$

where $I(t)$ is introduced to the master and the slave system to excite both systems as a continuous input.

In this paper, we will extend this approach to investigate if the slave system can anticipate the external source $I(t)$ and especially how the slave system anticipates the after-effect of a sudden change in the external source. Here, unexpected change of the dynamics does not come from the internal dynamics of the state variables $x$ or $y$, but from the data flow of the external source. 


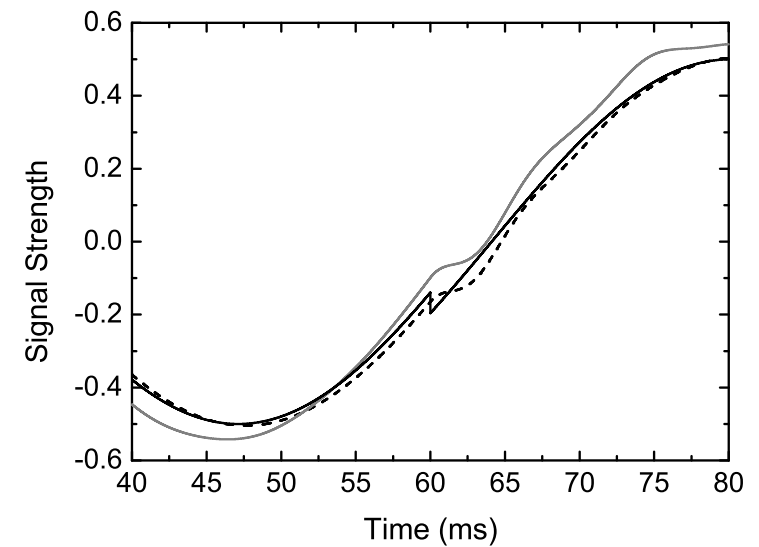

Fig. 1. Anticipatory synchronisation. The black line represents the external signal source, the gray line the slave system $y_{1}(t)$, and the dashed line the master system $x_{1}(t)$.

\section{MODELLING}

As a test model, we examine the coupled dynamics of a master and slave system described by a harmonic oscillator with a dissipative term driven by a periodic force term signal, $I(t)=A \sin (\omega t)$.

The slave receives time-delayed feedback of its own state variable in the coupling term with the master.

In the state space representation, the harmonic oscillator model for the master system is given by a system of two equations,

$$
\begin{aligned}
& \dot{x}_{1}=x_{2} \\
& \dot{x}_{2}=-\alpha x_{1}-\beta x_{2}+I(t),
\end{aligned}
$$

where $x_{1}$ and $x_{2}$ are state variables. For the slave system, we have similarly,

$$
\begin{aligned}
& \dot{y}_{1}=y_{2} \\
& \dot{y}_{2}=-\alpha y_{1}-\beta y_{2}+I(t)+k\left(x_{1}-y_{1, \tau}\right),
\end{aligned}
$$

where $y_{1}$ and $y_{2}$ are state variables and the last term represents a coupling between the master and slave systems. Note that the slave system incorporates the time delay, $y_{1, \tau}=$ $y_{1}(t-\tau)$ in the coupling term.

The selected parameters were; $A=0.5, \alpha=1.0, \beta=0.5$, $k=0.2, \omega=0.1 \mathrm{~Hz}, \tau=10 \mathrm{~ms}$. At the time of $60 \mathrm{~ms}$ the $\omega=0.1 \mathrm{~Hz}$ was shifted to $0.098 \mathrm{~Hz}$.

\section{RESULTS OF NUMERICAL CALCULATION AND DISCUSSION}

Fig. 1 shows the anticipatory synchronisation of the slave system $y_{1}(t)$ with respect to the external signal $I(t)$. Equations (6) and (8) show the constant precedence of the slave with respect to the external input, but the coupled systems are not simply phase-shifted as they are exposed to the periodic driving force. Rather, the introduction of the abrupt change in the external source clearly illustrates the anticipatory nature of the slave's response to such instantaneous changes and its ability to dynamically adjust to the changes in the external source dynamics. We also notice that the manifold $x(t)=y(t+\tau)$ is not fulfilled as $I(t)=I(t+\tau)$ is not satisfied.

These initial numerical results indicate that the external force might not have to be a harmonic oscillator. The coupled dynamical systems should show the anticipatory synchronisation as long as their dynamics are similar to the implicit dynamics of the external driver. It is natural that the coupled dynamics cannot anticipate the abrupt change itself, but they can respond quickly to the change in the external source.

Even though the anticipatory synchronisation itself is a robust phenomenon, in order to achieve a quantitative agreement between the slave system and the external source, the parameter space is restricted; for example, $k$ should be sufficiently small in order to ensure stability, and the frequency of the external oscillator should be sufficiently small compared to the characteristic frequency of the intrinsic dynamics in order to prevent phase lag between the slave and the master, and thus annihilating the effects of the slave's anticipation of the forcing term. The parameter space should be exploited substantially to obtain the phase diagram describing the stability of the anticipatory synchronisation.

Despite of these limitations, this is a first step to extend the strong anticipation paradigm to non-autonomous systems. As outlined above, this interesting phenomenon depends in interesting ways on the parameters involved and the configuration of the master, slave, and driving force couplings. The characterisation of these effects on strong anticipation will be the subject of subsequent studies.

\section{REFERENCES}

[1] D. A. Engström, J.A. S. Kelso, and T. Holroyd, Hum. Mov. Sci., 15, 809 (1996).

[2] B. H. Repp, Psychonomic Bulletin \& Review, 12-6, 969 (2005).

[3] F. Ishida and Y. Sawada, Phys. Rev. Lett., 96, 168105 (2004).

[4] Y. Hayashi, Y. Tamura, K. Sase, K. Sugawara, and Y. Sawada, Transactions of SICE, 46-7, 391 (2010).

[5] Y. Hayashi and Y. Sawada, Phys. Rev. E, in press.

[6] N. Stepp and M. T. Turvey, Cogn. Syst. Res. 11-2, 148 (2010).

[7] H. U. Voss, Phys. Rev. E, 61, 5115 (2000).

[8] M. Ciszak, O. Calvo, C. Masoller, C. Mirasso, and R. Toral, Phys. Rev. Lett. 90, 204102 (2003). 